
Risk Rankings

Michiel C.W. van de Leur

André Lucas 
Tinbergen Institute is the graduate school and research institute in economics of Erasmus University Rotterdam, the University of Amsterdam and VU University Amsterdam.

More TI discussion papers can be downloaded at http://www.tinbergen.nl

Tinbergen Institute has two locations:

Tinbergen Institute Amsterdam

Gustav Mahlerplein 117

1082 MS Amsterdam

The Netherlands

Tel.: +31(0)20525 1600

Tinbergen Institute Rotterdam

Burg. Oudlaan 50

3062 PA Rotterdam

The Netherlands

Tel.: +31(0)10 4088900

Fax: +31(0)10 4089031 


\title{
Network, Market, and Book-Based Systemic Risk Rankings ${ }^{\text {th }}$
}

\author{
Michiel C.W. van de Leur ${ }^{\mathrm{a}}$, André Lucas ${ }^{\mathrm{a}}$ \\ ${ }^{a}$ Vrije Universiteit Amsterdam and Tinbergen Institute
}

\begin{abstract}
We investigate the information content of stock correlation based network measures for systemic risk rankings, such as SIFIRank (based on Google's PageRank). Using European banking data, we first show that SIFIRank is empirically equivalent to a ranking based on average pairwise stock correlations. Next, we find that correlation based network measures still appear to complement currently available systemic risk ranking methods based on book or market values. A further analytical investigation, however, shows that the value-added appears to be mainly attributable to pairwise cross-sectional heterogeneity rather than to more subtle network relations and feedback loops.
\end{abstract}

Keywords: Systemically Important Financial Institutions (SIFI), European banking sector, systemic risk rankings, network based risk measures.

JEL: G01, G21

\section{Introduction}

The 2008 financial crisis and subsequent European sovereign debt crisis have led to a continuing interest in how to allocate systemic risk ${ }^{1}$ over firms that make up the financial system; see Tarashev et al. (2010). Systemic risk allocation has, for example, been embedded into formal regulation by labeling some financials as Systemically Important Financial Institutions (SIFIs) subject to increased capital requirements. Different methods have been put forward to distinguish SIFIs from non-SIFIs using so-called systemic risk rankings; see Benoit et al. (2015) for a recent survey. Most of these ranking methodologies make use of readily available data and simple statistical measures, such stock betas, volatilities, (tail) dependence measures, (conditional) values-at-risk, etc.

Dungey et al. (2013) propose a different ranking method based on network concepts. More specifically, their SIFIRank approach directly builds on the Google PageRank methodology. It does not only take into account the pairwise correlations between (financial) firms, but also the indirect linkages and feedback loops that operate in the system as a whole. The method can be extended by including familiar firm characteristics such as size, leverage and liquidity. The appeal of the PageRank methodology of Dungey et al. (2013) is that it uses a network philosophy for ranking without having to use proprietary data sources such as balance sheet positions, cross-holdings, or interbank payments. This makes the PageRank methodology much easier to implement on readily available data sets than alternatives that require specialized or proprietary data, such as Craig and von Peter (2014), Anand et al. (2015), Brauning and Fecht (2012), and Abbassi et al. (2015)

Despite their popularity to track financial interconnectedness, it is still unclear whether network based ranking measures add significantly to simpler measures, such as Marginal Expected Shortfall (MES) of Acharya et al. (2010), $\Delta$ CoVaR of Adrian and Brunnermeier (2014) and Castro and Ferrari (2014), or

\footnotetext{
We thank participants of the SYRTO seminar for their helpful comments and useful suggestions and comments. We thank the European Union Seventh Framework Programme (FP7-SSH/2007-2013, grant agreement 320270 - SYRTO) for financial support.

Email addresses: m.c.w.vandeleur@uva.nl (Michiel C.W. van de Leur), a.lucas@vu.nl (André Lucas)

${ }^{1}$ For surveys on systemic risk research, see for example ECB $(2009,2010,2011)$ and Bisias et al. (2012).
} 
SRISK of Brownlees and Engle (2010). These simpler measures are based on pairwise relationships only, either between two stocks or between a stock and an index. More specifically, these measures do not take the whole interconnectedness of the financial network into account.

In this paper we address the question whether systemic risk rankings that use correlation based network measures succeed in capturing substantially different aspects of systemic risk than simple book or market based rankings. We do so in two different ways. First, we compare the network based measures based on the Google PageRank methodology to a new, simple method based on the average pairwise correlations. We call the latter method CorrRank. As opposed to PageRank, CorrRank does not take feedback loops within the financial system into account. Perhaps surprisingly, the correspondence between the two ranking methodologies is high, irrespectively of whether we consider the period before, during, or after the financial crisis. Hence, the first order systemic risk effects based on stock correlation matrices appear to be mainly driven by direct rather than indirect linkages.

Second, we compare different systemic risk rankings using a principal components analysis, similar to the analyses in Billio et al. (2012), Moreno and Pena (2013), Giglio et al. (2015), and Nucera et al. (2015). In contrast to previous work, we focus on the value-added of the network based measures compared to the most popular non-network based alternatives. We use the analysis of Benoit et al. (2013) to reduce the number of relevant alternatives to two: beta times the market value, and value at risk (or volatility). Benoit et al. (2013) show analytically that in a stylized setting the most popular risk ranking measures (MES, SES, SRISK and $\triangle \mathrm{CoVaR}$ ) can be characterised by beta and Value-at-Risk (VaR); see also Adams et al. (2014) and White et al. (2015). Based on their arguments, Benoit et al. also show empirically that for instance beta and MES reveal nearly equivalent dynamic patterns of systemic risk, as predicted by their theoretical analysis. Also Nucera et al. (2015) find that only two components (one book-based and one market-based) out of a range of available systemic risk ranking methodologies suffice to summarize most of the cross-sectional and time series variation in these rankings.

To test the value-added of network based measures, we use a sample consisting of the S\&P Europe 350 . We augment the sample by a number of large European banks to obtain a total of 119 financial and 282 non-financial firms over the period October 1995 until October 2015. Using different ranking methods, we determine the systemic risk ranks of each firm for each time period. Based on the individual firm ranks, we also determine the systemic risk ranking of the financial sector as a whole. We find that the overall systemic risk rank of the financial sector shifts substantially over the different crises periods in the sample. In addition, the different ranking methodologies do not move in tandem over time. This is confirmed by our principal components analysis, which shows that the first two principal components explain roughly $85 \%$ of the rank variation. The first component coincides with the average rank across all ranking methods. The second component, however, clearly distinguishes between the information contained in the network based rankings versus the more familiar book-based and market-based rankings.

Next, we zoom in on the analytic origins of the different time series patterns of alternative ranking methodologies. We find that network based rankings such as PageRank and CorrRank mainly differ from beta or market value based rankings due to cross-sectional heterogeneity of pairwise stock correlations, rather than to higher order subtle network relationships and possible feedback loops. This leads to two main implications. First, systemic risk rankings (such as MES or SRISK) that only use the correlation between a stock and an index may miss a substantial part of information compared to measures that take all pairwise correlations into account (such as CorrRank). Second, despite their ready availability, network measures based on simple stock correlations (such as PageRank or CorrRank) may not suffice to uncover new systemic risk features beyond pairwise heterogeneity. To improve further, new information is required from other sources, such as proprietary cross-holdings or payment systems information. Only such additional information can provide a complementary view to simple (pairwise) correlations, betas, book values, and volatilities as to what constitutes the systemically important firms in the financial system.

The rest of this paper is set up as follows. Section 2 introduces the different systemic risk ranking methods. The data and results are described in Section 3. Section 4 concludes. 


\section{Systemic risk rankings}

\subsection{Network inspired ranking methods}

The network structure of the financial system received more and more attention over the past years, both theoretically and empirically. ${ }^{2}$ A network structure may add information to commonly used pairwise interconnections by including indirect effects as well as higher order feedback loops in determining the vulnerability of the system as a whole. This is particularly important if there are possible cascade effects via indirect linkages of financial institutions, either via the asset side, or the liability side.

Many of the network based measures suggested in the literature suffer from two main drawbacks. First, they often require proprietary data to be operationalized. In particular, bank network measures typically use mutual cross-exposures of financial institutions. This information is available for regulators, but not for the public at large. Alternatively, partial cross-exposures may be inferred by keeping track of repeated bilateral transactions between financial institutions and backing out the cumulative positions of both parties. This is for instance done using payments data; see for instance Brauning and Fecht (2012). Though such information becomes increasingly accessible, the sensitive nature of the transactions data involved still restricts their use to a limited number of researchers. A second main drawback of network measures is that they typically concentrate on the financial system as a self-contained subsystem in the wider economy. While this still allows for the construction of both marginal contribution measures to systemic risk by individual institutions as well as for concentration measures of the financial system as a whole, it does not allow one to determine the importance of the financial system vis-à-vis the real economy.

\section{PageRank}

An important measure that overcomes both of these drawbacks is introduced by Dungey et al. (2013). They use ranking methods based on the Google PageRank methodology. The approach has four main advantages. First, the method retains the network based flavor of other network methods that require proprietary data. In particular, it does not only account for pairwise linkages between firms, but also for higher order interconnections. It does so by concentrating on the eigenvector corresponding to the matrix of pairwise connection strengths. Second, the method can be based on readily available public data, in particular stock price returns. The main ingredient of the method is the matrix of pairwise connection strengths, which is taken to be the time varying estimate of the correlation matrix. Third, being based on readily available stock return data, the method easily allows for the inclusion of both financial and non-financial firms in the network. As a result, the method can be used to determine both the level of concentration inside the network and the relative importance of the financial vis-à-vis the non-financial sector in the network. Finally, the method allows for the inclusion of additional, exogenous information in the systemic risk rankings. Important candidate variables are size, leverage, and illiquidity of the firm.

More concretely, the central piece of information in Dungey et al. (2013) is the time $t$ estimate of the normalized correlation matrix, denoted as $\widehat{\boldsymbol{C}}_{t} \in \mathbb{R}^{n \times n}$, where $n$ is the number of firms. The $(i, j)$ th element $\hat{c}_{i, j, t}$ of $\widehat{\boldsymbol{C}}_{t}$ equals

$$
\hat{c}_{i, j, t}=\hat{\rho}_{i, j, t} / \sum_{k \neq j} \hat{\rho}_{k, j, t},
$$

for $i \neq j$, and $\hat{c}_{i, i, t}=0$, where $\hat{\rho}_{i, j, t}$ is the estimate of the pairwise correlation between firm $i$ and firm $j$ at time $t$ based on a rolling window of 200 days. Given the matrix $\widehat{\boldsymbol{C}}_{t}$, PageRank determines the vector $\boldsymbol{S}_{t} \in \mathbb{R}^{n \times 1}$ as the eigenvector of $\widehat{\boldsymbol{C}}_{t}$ corresponding to the unit eigenvalue, i.e.,

$$
\boldsymbol{S}_{t}=\widehat{\boldsymbol{C}}_{t} \boldsymbol{S}_{t}
$$

The elements in the vector $\boldsymbol{S}_{t}$ indicate the systemic influence of each firm on the system as a whole. A unit shock $\boldsymbol{S}_{j, t}$ to firm $j$ th is spread out over the other nodes using the normalized correlations as weights. The

\footnotetext{
${ }^{2}$ See for example for a theoretical perspective Georg (2013), Acemoglu et al. (2015), Afonso and Lagos (2015), and Glasserman and Young (2015). For an empirical perspective, see Brauning and Fecht (2012), Abbassi et al. (2015), Craig and von Peter (2014), and Anand et al. (2015).
} 
resulting dispersed second round shock is fed back into the system until the whole system is in equilibrium. The resulting $\boldsymbol{S}_{t}$ is used to compute the systemic risk rankings, with a high rank of $\boldsymbol{S}_{j, t}$ in $\boldsymbol{S}_{t}$ indicating a systemically important firm.

The PageRank method can be further refined by including firm characteristics that could be important for determining a firm's systemic importance. Typical examples of such variables are firm size, leverage, and illiquidity. When included into PageRank, these characteristics are first normalized by the sum of the variable over all firms. The eigenvector problem is then modified into

$$
\boldsymbol{S}_{t}=\alpha \cdot \widehat{\boldsymbol{C}}_{t} \boldsymbol{S}_{t}+\left(\text { Size }_{t}, \text { Leverage }_{t}, \text { Illiquidity }_{t}\right) \gamma
$$

where Size $_{t}$, Leverage $_{t}$, Illiquidity $_{t} \in \mathbb{R}^{n \times 1}$ are the vectors with firm characteristics (expressed as percentages) log market capitalization, log leverage, and Ammihud's stock illiquidity measure, respectively, and where $\alpha$ and $\gamma$ are fixed, predetermined coefficients. As $\alpha$ and $\gamma$ are not empirically identified, Dungey et al. (2013) propose to set $\alpha=0.66$ and $\gamma^{\prime}=(0.4,0.4,0.2)$. The elements of $\boldsymbol{S}_{t}$ of this modified eigenvector problem are again ranked to obtain the adjusted PageRank*.

\section{CorrRank}

The PageRank method does not only take the pairwise correlations of a firm with other firms into account, but also the indirect spillovers of one firm to all other firms in the system. It does so by the eigenvector methodology described above. The importance of such second order effects in typical economic data, however, is debatable. We therefore also introduce a simpler measure of interconnectedness of firm $i$ with the rest of the system by defining the CorrRank measure. CorrRank determines the cross-sectional rank of firm $i$ in terms of $\sum_{k} \rho_{i, k, t}=\sum_{k} \rho_{k, i, t}$. It only accounts for the total pairwise correlations without any second order effects. It allows us to answer to what extent other network risk measures such as PageRank also incorporate the subtler aspects of the network structure. In network terms, CorrRank captures the weighted out-degree ${ }^{3}$ of every node, where weights are assigned based on the correlation values.

\subsection{Other ranking methods}

As mentioned in the introduction, there is by now a large number of alternative systemic risk ranking methodologies. For example, Giglio et al. (2015) compare 19 different systemic risk rankings via a principal components analysis. Interestingly, Benoit et al. (2015) compare a number of these methodologies in a simplified analytical framework. They show that under specific conditions the Marginal Expected Shortfall (MES) of a firm as introduced by Acharya et al. (2010) is proportional to its beta multiplied by the expected shortfall of the market. As the expected shortfall of the market is common to all firms, MES and beta should lead to similar systemic risk rankings. Analogously, Benoit et al. (2015) show that the $\Delta$ CoVaR of Adrian and Brunnermeier (2014) and Castro and Ferrari (2014)) is a linear transformation of the Value-at-Risk of Adams et al. (2014) and White et al. (2015), where the coefficients in the linear relation are common across all firms. This reduces a large number of familiar risk ranking methods to a much smaller number, namely the firm's beta and its VaR. These findings are confirmed by the empirical analysis in Nucera et al. (2015), who find that a combination of a book based ranking and a market-value based ranking reflects most of the cross-sectional and time-series variation in available systemic risk rankings. In the remainder of this paper, we therefore restrict our attention to a beta and a VaR related ranking methodology as alternatives to the network based rankings described earlier.

\section{BetaRank}

BetaRank measures the rank of a firm based on its systematic rather than systemic risk. The beta is computed by regressing stock market returns on the S\&P Europe 350 index. Following Nucera et al. (2015), we weight the beta by the market value of the firm to obtain a measure of systematic capital at risk. The betas are estimated using a rolling window of the previous 200 trading days. Compared to PageRank or CorrRank, BetaRank only measures the relation of the firm to the market as whole, multiplied by its own

\footnotetext{
${ }^{3}$ Due to the symmetry of the correlation matrix, this is also the weighted in-degree.
} 
size, and abstracts from all pairwise interconnections. For the sample of large cap firms we consider in our empirical study in Section 3, ranks based on beta or on beta times market value are highly similar. ${ }^{4}$

\section{VaRRank}

VaRRank is based on a firm's Value-at-Risk. Hence, it only reflects the riskiness of the firm itself. In comparison with other methods it does not consider the correlation or covariance with either the market as a whole or with specific other firms. We compute VaRRank based on the $95 \%$ daily VaR, which we compute nonparametrically by the 10th worst return observed over the (rolling) past 200 trading days. This return is multiplied by the market capitalization of the firm to obtain the VaR.

The five ranking methods above (PageRank, PageRank*, CorrRank, BetaRank, VaRRank) give a broad, yet concise overview of the different raking methodologies proposed in the literature. The methods comprise both the market and book based methodologies which were classified as most important by Benoit et al. (2015) and Nucera et al. (2015). At the same time, the network based method of Dungey et al. (2013) is included, which allows us to investigate the value-added of network based ranking methodologies.

\section{Results}

\subsection{Data}

In our analysis, and following Dungey et al. (2013), we consider both the systemic risk rankings of individual institutions, as well as the average systemic risk ranking of the financial sector as a whole vis-àvis the non-financial sector. We take the constituents of the S\&P Europe 350 index in 2015 as a reflection of the broad economic activity in Europe. ${ }^{5}$ We add the 54 largest European banks that are not included in the index in order to obtain a broader reflection of the European financial sector. For all firms in our sample, we take daily stock returns from Datastream for a 20-year period from Oct 2, 1995 until Oct 2, 2015. For every day in our sample we compute our systemic risk rankings based on parameter estimates of a rolling 200 day window. The sample contains a maximum of 401 firms at any moment in time, with a maximum of 119 financial firms.

To construct the PageRank measure of Dungey et al. (2013), we follow their approach and also download Size, Leverage and Liquidity for each firm in the sample. Size is measured as the book value of total assets; Leverage is the ratio of total book value of debt over market value of equity; and Illiquidity is measured as the inverse of liquidity, where liquidity is the ratio of net cash over total assets. The definitions are in line with Dungey et al. (2013). ${ }^{6}$

As a first analysis, we rank firms on the pure firm characteristics size, leverage, and illiquidity. We use these ranks to compute the average rank of financial versus non-financial firms in our sample. Figure 1 plots the result. A higher value in the figure means a higher size, leverage, or illiquidity. Because the number of firms is not constant over time, we follow Nucera et al. (2015) and scale the ranks by the number of firms present at time $t$. The scaled ranks now all lie within the unit interval. If financials are just as systemically important as non-financials, we expect a financial sector average rank of 0.5. It is clear that the average size, leverage, or illiquidity rank of financial sector is larger than for the non-financial sector. The values plotted in Figure 1 always exceed 0.5. We see a decline in the leverage rank of the financial sector over the late 1990 and early 2000s, and a subsequent gradual increase over the entire period since 2002. The leverage ranks do not come down substantially during the financial crisis, nor during the subsequent European sovereign debt crisis.

\footnotetext{
${ }^{4}$ The rank correlations between the two measures is 0.935 .

${ }^{5}$ Given that we add the 54 largest banks to our sample of firms, we argue that the effect of any possible survivorship bias due to the selection of the 2015 constituents is mild. In particular, we expect this to have little effect on the average rank of the financial system compared to the non-financial sector, as well as on the order of ranks within the financial system.

${ }^{6}$ More precisely, total assets equals the sum of total current assets, long term receivables, investment in unconsolidated subsidiaries, other investments, net property plant and equipment and other assets. Illiquidity is defined as $l i q_{k, t}^{-1}=$ $\log \mid$ Liquidity $_{k, t}-1-\max _{j}$ Liquidity $_{j, t} \mid$

$\log \left(1+\max _{j}\right.$ Liquidity $\left._{j, t}\right)$
} 


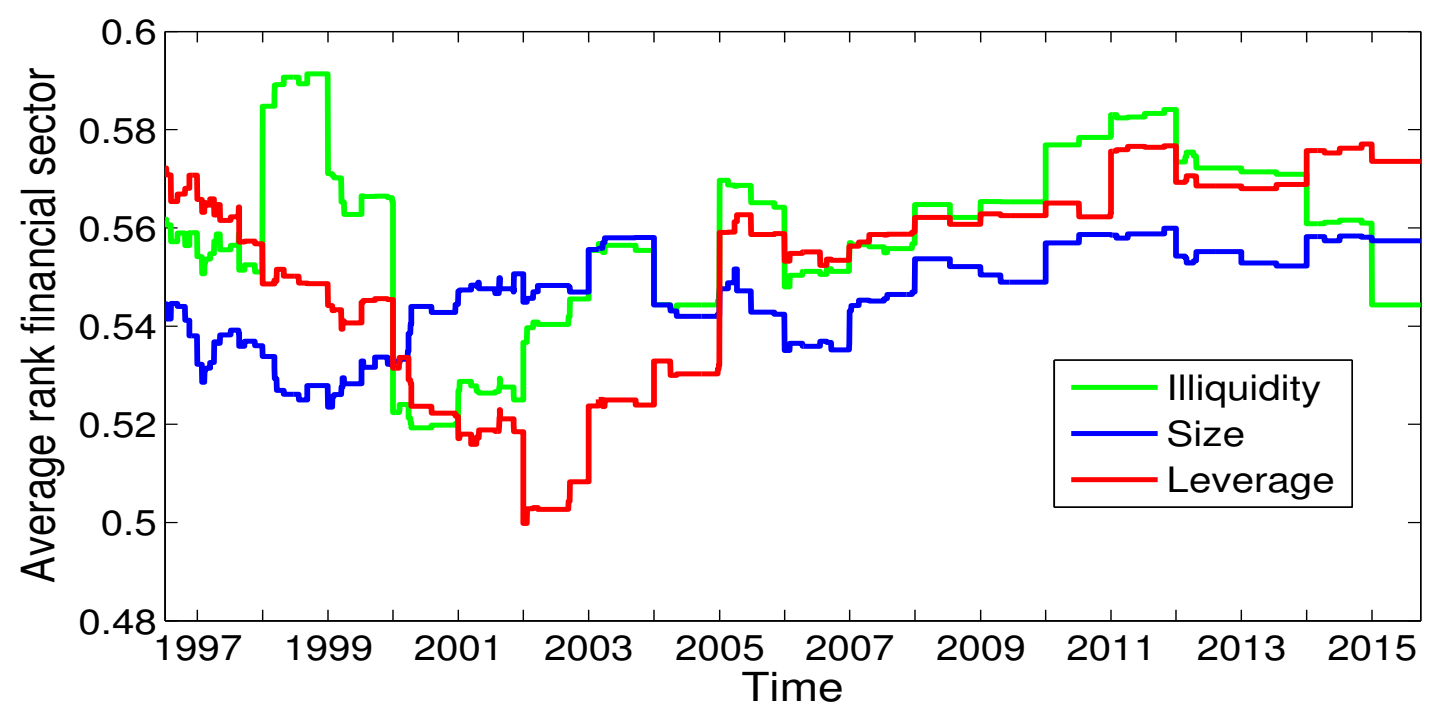

Figure 1: Average SizeRank, IlliquidityRank, and LeverageRank of the European financial sector; higher values imply higher size, leverage, or illiquidity.

\subsection{Information in PageRank versus CorrRank}

In the first part of our analysis, we investigate the differences between PageRank versus the simpler CorrRank measure. In this way, we can see whether the direct linkages (as embedded in CorrRank) contain all information for systemic risk rankings, or whether there is additional information contained in the more subtler network structure, i.e., in the indirect linkages (as embedded in PageRank).

Figure 2 plots the average financial sector rank over time for CorrRank, PageRank, and PageRank*. In the figure, PageRank and CorrRank are almost indistinguishable. The correlation of CorrRank with PageRank is 0.9998. The addition of the firm characteristics to the rankings as done in PageRank* does not substantially alter the picture. The overall pattern remains similar, and the absolute differences are small, particularly after 2006. The correlation between CorrRank and PageRank* is again high: 0.9814. Hence the extra information exploited in PageRank compared to CorrRank appears negligibly for the data at hand: it appears we can rank the financial sector systemic risk based on the average pairwise correlations alone.

\subsection{CorrRank versus BetaRank and VaRRank}

In the previous subsection we concluded that the network-based measures can be simplified to the CorrRank measure. Hence in the remainder of the analysis we will only use the CorrRank and compare it to VaRRank and BetaRank. The results are shown in Figure 3. The time series correlations for the three ranking methods are presented in Table 1 . These have to be interpreted with care, as the pattern in Figure 3 shows that the time series are probably non-stationary. We therefore also plot the correlations of the first differenced time series. Particularly looking at the correlations of the first differences, we see that the information in each of the series is overlapping, but that the overlap is far from perfect. The highest correlation is 0.409 and is between VaRRank and CorrRank. ${ }^{7}$

Whereas Table 1 gives the time series correlation between different ranking methods of the cross-sectional average of the financial sector rank, Figure 4 plots the time series of the pairwise cross-sectional rank correlations. The cross-sectional correlations come closest to our principal components analysis later on that

\footnotetext{
${ }^{7}$ Figure 3 plots the average rank of the financial industry. If we plot the median rank instead, the picture hardly changes, suggesting that the rankings of financials are not highly skewed.
} 


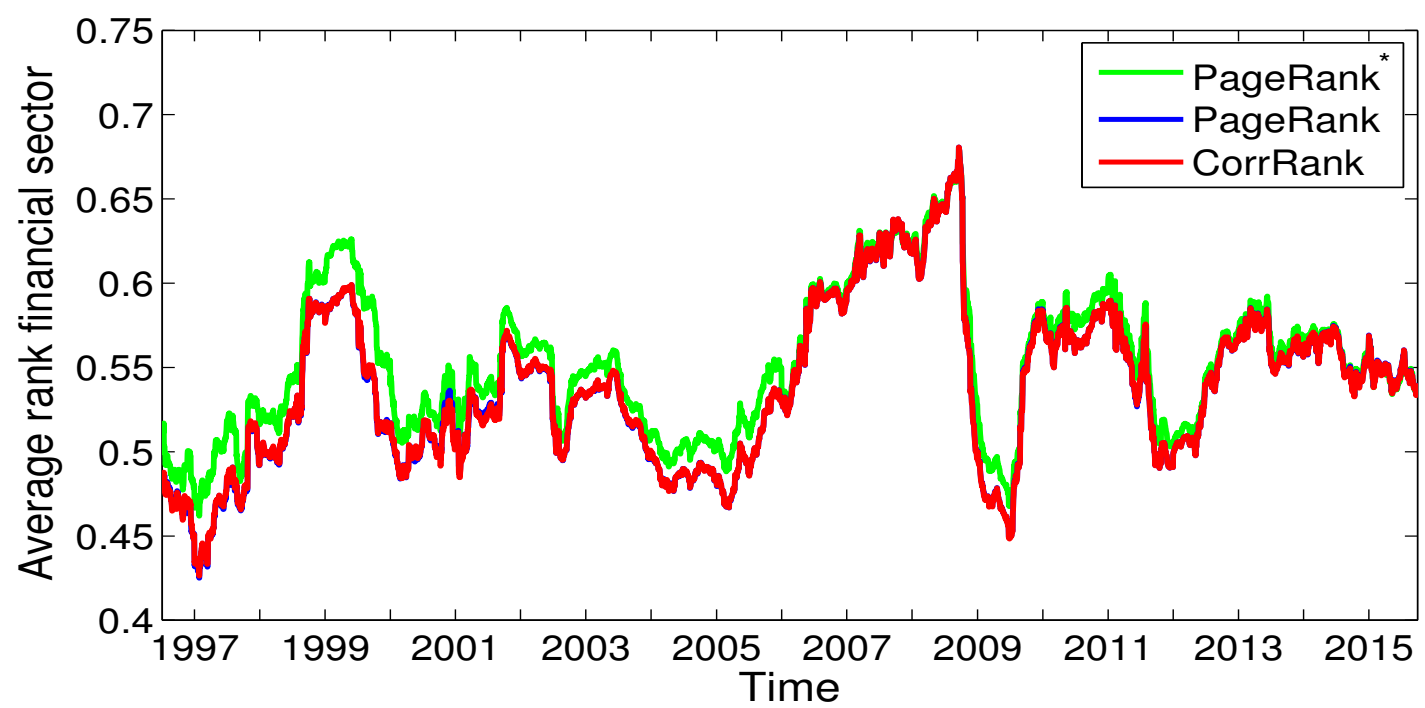

Figure 2: Comparison average financial sector score of three correlation based rankings

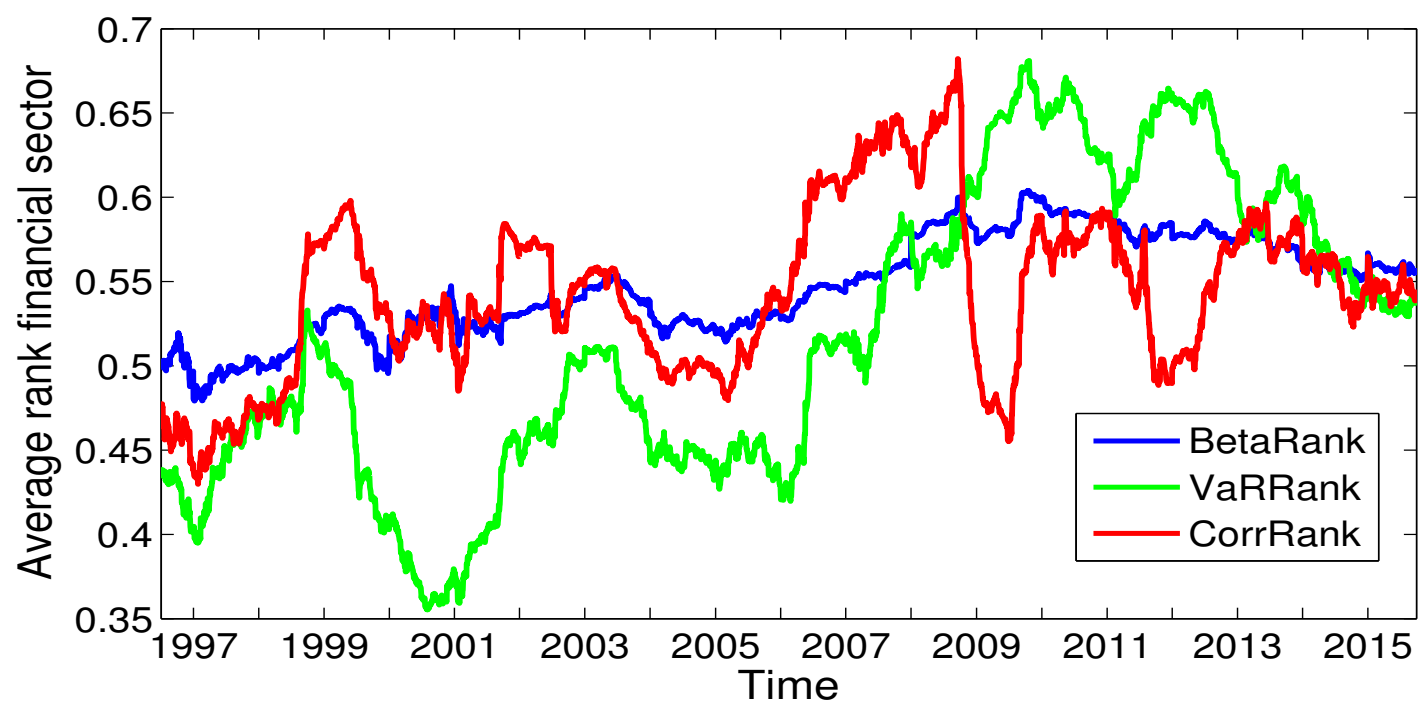

Figure 3: Comparison of total financial sector systemic rank for three main rankings.

we use to determine which ranking methods contain the most information. We perform the analysis for the entire universe of stocks, but the results are highly similar if restricted to the subsample of financial stocks. We observe the highest (positive) correlation between the VaRRank and BetaRank. The correlation remains rather constant throughout the sample. By contrast, we observe that CorrRank is largely positively correlated with both VaRRank and BetaRank up till 2006, but that after this period, the correlation breaks to a much lower level and becomes zero or even slightly negative. Hence, where BetaRank and VaRRank to large extent appear to tell a similar story, the CorrRank measure appears to contain different information about the importance of the financial sector. 
Table 1: Correlation matrix of different systemic risk ranking methods

\begin{tabular}{llllllll}
\hline & \multicolumn{3}{c}{ Levels } & & \multicolumn{3}{c}{ 1st differences } \\
\cline { 2 - 3 } & CorrRank & BetaRank & VaRRank & & CorrRank & BetaRank & VaRRank \\
\hline CorrRank & 1 & 0.542 & 0.336 & & 1 & 0.201 & 0.409 \\
BetaRank & 0.542 & 1 & 0.881 & & 0.201 & 1 & 0.240 \\
VaRRank & 0.336 & 0.881 & 1 & & 0.409 & 0.240 & 1 \\
\hline
\end{tabular}

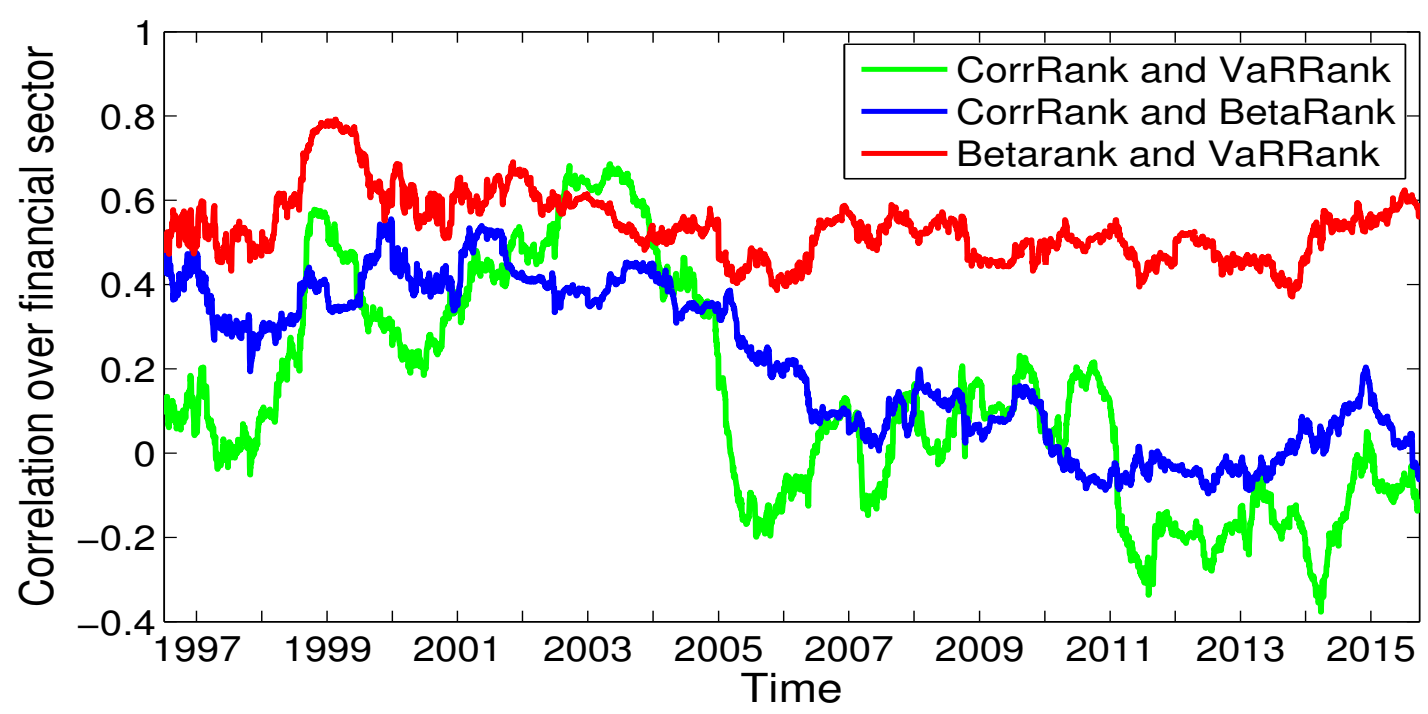

Figure 4: Pairwise correlations

This is confirmed when we look at the results of our Principal Component Analysis on CorrRank, VaRRank, and BetaRank in Figures 5 and 6. We observe in Figure 5 that the first principal component explains on average $56 \%$ of the cross-sectional rank variation. The first two components together even explain $85 \%$ of the variation. The latter percentage is highly stable over the entire sample period, which can be seen by the stability of the third principal component's contribution.

To understand what are the information sources making up the first two principal components, we look at the factors and the factor loadings over time. These are plotted in Figure 6. There are two episodes where the two factors clearly convey a different message: the late 1990s combined with the burst of the dotcom bubble early 2000, and the financial crisis combined with the subsequent sovereign debt crisis (see panel (a)). Looking at the factors together with the different ranking methods (panel (b)), it appears that the first component mainly follows the overall pattern of the three combined rankings. The second component mainly appears to pick up on the aberrant behavior of some of the rankings during periods of stress: mainly the VaRRank and CorrRank during the dotcom crash, and mainly CorrRank during the financial crisis.

We obtain further insight by looking at the loadings of the first two components in panels (c) and (d), respectively. The loadings for the first principal component (panel (c)) appear to be roughly equally sized up to 2005, with a somewhat smaller weight for CorrRank. After 2005, the loading for CorrRank drops repeatedly over the rest of the sample period in order to end up near 0 at the end of the sovereign debt crisis. This result suggests that the network based systemic risk ranks CorrRank and PageRank reflect different information than the combination of BetaRank and VaRRank. The pattern is confirmed by the loadings on the second principal component in panel (d). The second component mainly loads on the CorrRank measure. BetaRank and VaRRank receive a cumulative weight of around -0.5 , except towards the end of the sample 


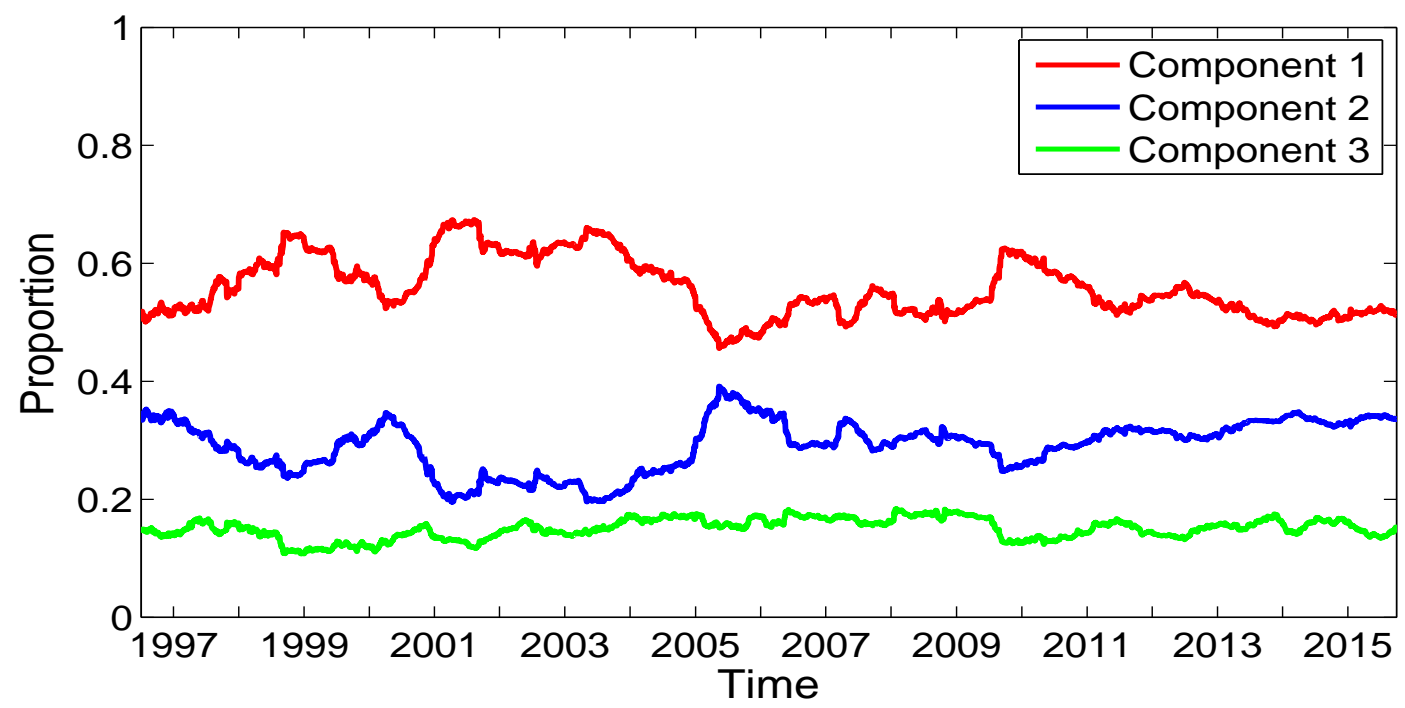

Figure 5: Explained proportion by each principal component (summing to 1)

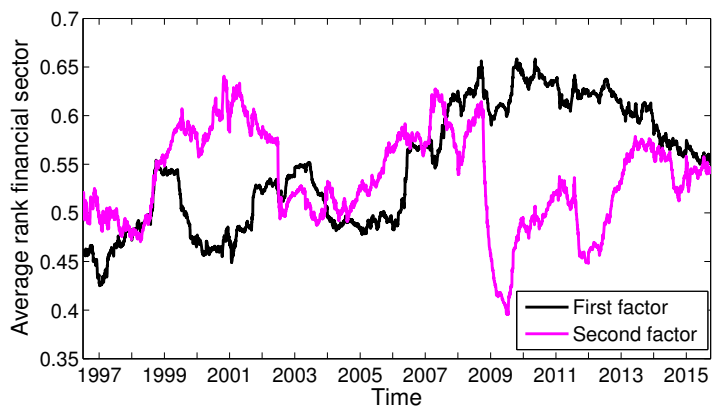

(a) Factors.

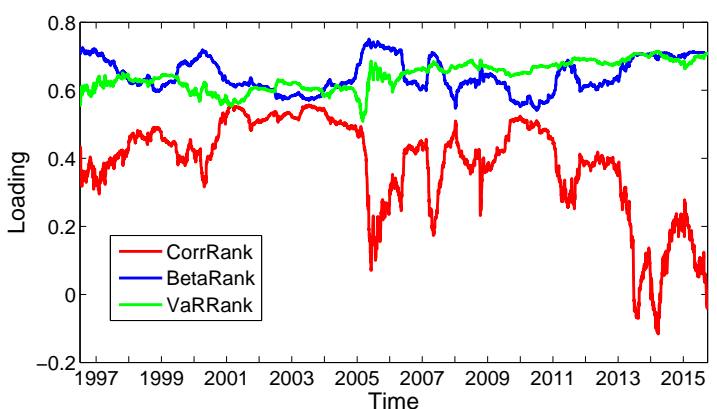

(c) Loadings 1st principal component.

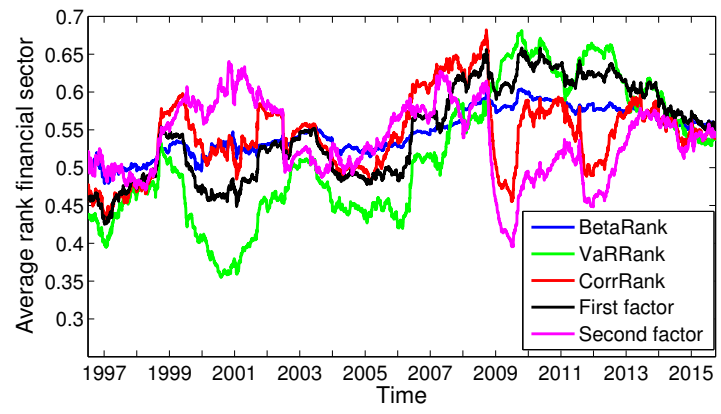

(b) Factors and Rankings.

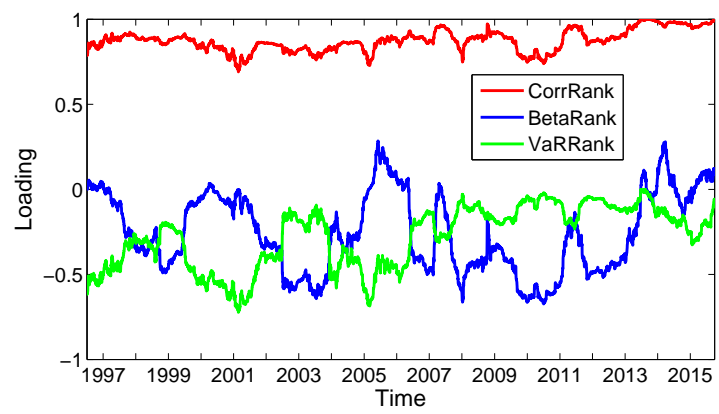

(d) Loadings 2nd principal component.

Figure 6: First two principal components and their loadings

where they receive no weight. Again, the results show that the network based CorrRank measure contains valuable information for systemic risk ranking beyond and above the familiar BetaRank and VaRRank. 
As the latter two measures are taken as representatives of a wider class of systemic risk ranking methods following the arguments in Benoit et al. (2015) and Nucera et al. (2015), we conclude that network based measures such as PageRank and its current equivalent CorrRank (see Figure 2) contain additional valuable information compared to other available ranking methods.

\subsection{Information differences: network properties or heterogeneity}

To further understand what information is contained in PageRank and CorrRank that is not incorporated in the combination of VaRRank and BetaRank, we outline a decomposition of CorrRank to relate it more closely to BetaRank. The key quantity in the BetaRank measure is the firm's beta times its market value. This quantity is given by

$$
\beta_{i, t} \times M V_{i, t}=\frac{\operatorname{cov}\left(R_{i, t}, R_{t}^{m}\right)}{\operatorname{var}\left(R_{t}^{m}\right)} \times M V_{i, t}=\frac{\sum_{j \in \mathcal{I}} w_{j, t} \operatorname{cov}\left(R_{i, t}, R_{j, t}\right)}{\operatorname{var}\left(R_{t}^{m}\right)} \times M V_{i, t},
$$

where $R_{i, t}$ is the excess return on stock $i$ over period $t, R_{t}^{m}$ is the market excess return, $\mathcal{I}$ denotes the set of stocks in the index, and $w_{i, t}=M V_{i, t} / \sum_{j \in I} M V_{j, t}$ is the market capitalization weight of the stock in the index. We can further rewrite (1) as

$$
\beta_{i, t} \times M V_{i, t}=\left(\sum_{j \in \mathcal{I}} w_{j, t} \frac{\sigma_{i, t} \sigma_{j, t}}{\sigma_{m, t}^{2}} \rho_{i, j, t}\right) \times M V_{i, t}
$$

where $\rho_{i, j, t}$ denotes the correlation between stocks $i$ and $j$ at time $t$, and $\sigma_{i, t}, \sigma_{j, t}$, and $\sigma_{m, t}$ denote the standard deviations for stock $i$, stock $j$, and the market index at time $t$, respectively.

The key quantity determining CorrRank is

$$
\left(\sum_{j \in \mathcal{S}} \frac{1}{|\mathcal{S}|} \rho_{i, j, t}\right)
$$

where $\mathcal{S}$ is the sample of stocks considered in the analysis, containing $|\mathcal{S}|$ elements. The different information contained in CorrRank versus BetaRank thus stems from three main sources: (i) the difference in the universe of stocks $\mathcal{S}$ making up the sample versus the universe of stocks $\mathcal{I}$ making up the index; (ii) the use of an equal weighted sum of the pairwise correlations (with weights $1 /|\mathcal{S}|$ ) in CorrRank versus a weighted sum (with weights $w_{j, t} \sigma_{i, t} \sigma_{j, t} / \sigma_{m, t}^{2}$ ) in BetaRank; and (iii) the multiplication by the market capitalization of the firm. The information in BetaRank and CorrRank thus becomes more similar if the index return is based on the same sample of firms as the correlation matrix; an equally weighted rather than a capitalization weighted index would be used; time-varying volatilities would be driven by a single common factor; and the weighting by the market capitalization $M V_{i, t}$ would be omitted. Interestingly, none of these features is directly linked to the higher order linkages in the network of firms. Rather, the difference in information between CorrRank and VaRRank appears to be due to the heterogeneity of the individual stock attributes, and the possible difference between the stocks making up the index versus the stocks in the sample. This finding has an important implication. Network based systemic risk measurement systems appear to require investments in high-quality new data sources, such as (possibly confidential) cross-holding and cross-exposure data. Cheap alternatives based on pairwise correlations and their implied network structure appear to add hardly any information.

\section{Conclusions}

In this paper we have compared different ranking methods for determining what are Systemically Important Financial Institutions. In particular, we have investigated whether the Google PageRank methodology based on a pairwise correlation matrix adds value to more familiar ranking methodologies based on stock betas or Values-at-Risk (VaRs). Using a sample of European stocks combined with the largest European 
banks, we found that the PageRank measure contained the same information as a much simpler measure based on average pairwise correlations (CorrRank). Still, using a principal components perspective, the PageRank (or CorrRank) measure appeared to contain additional cross-sectional information compared to ranks based on betas or VaRs. The difference in information, however, appeared to be due to cross-sectional heterogeneity in stock attributes (such as market capitalization, time varying stock return volatility) rather than to subtleties in the network structure and the occurrence of possible feedback loops in the network.

We derive two main implications from our analysis. First, currently used measures that only exploit the correlation between a financial stock return and an index may miss out on the cross-sectional (and possibly time varying) heterogeneity of pairwise relationships. Measures such as PageRank and CorrRank may therefore add information aspects to currently used measures. Second, we conclude that you get what you pay for. Cheap alternatives to construct network structures to assess systemic risk appear only moderately useful. The current network measures based on pairwise stock return correlations are easy to construct on readily available data, but appear to add little to simpler measures based only on pairwise relations such as CorrRank. This suggests that the value-added of network measures should go hand-in-hand with the use of new, possibly proprietary data sources. Examples of this include the use of payments data or crossexposure data, as it is becoming more and more available particularly at regulatory institutions. It would be interesting to include such rankings based on more elaborate data sources in future comparisons.

\section{References}

Abbassi, P., Bräuning, F., Fecht, F., and Peydró, J.-L. (2015). Cross-border liquidity, relationships and monetary policy: Evidence from the euro area interbank crisis. CEPR Discussion Papers, 10479.

Acemoglu, D., Ozdaglar, A., and Tahbaz-Salehi, A. (2015). Systemic risk and stability in financial networks. American Economic Review, 105(2):564-608.

Acharya, V., Pedersen, L., Philippon, T., and Richardson, M. (2010). Measuring systemic risk. NYU workingpaper.

Adams, Z., Füss, R., and Gropp, R. (2014). Spillover effects among financial institutions: A state-dependent sensitivity valueat-risk approach. Journal of Financial and Quantitative Analysis, 49:575-598.

Adrian, T. and Brunnermeier, M. (2014). CoVaR. Federal Reserve Bank of New York Staff Reports, 348.

Afonso, G. and Lagos, R. (2015). Trade dynamics in the market for federal funds. Econometrica, 83(1):263-313.

Anand, K., Craig, B., and von Peter, G. (2015). Filling in the blanks: network structure and interbank contagion. Quantitative Finance, 15:625-636.

Benoit, S., Colletaz, G., Hurlin, C., and Pérignon, C. (2013). A theoretical and empirical comparison of systemic risk measures. Working paper.

Benoit, S., Colliard, J.-E., Hurlin, C., and Pérignon, C. (2015). Where the risks lie: A survey on systemic risk. Working paper.

Billio, M., Getmansky, M., Lo, A., and Pellizzon, L. (2012). Econometric measures of systemic risk in the finance and insurance sectors. Journal of Financial Economics, 104:535-559.

Bisias, D., Flood, M., Lo, A., and Valavanis, S. (2012). A survey of systemic risk analytics. Office of Financial Research working paper, 1.

Brauning, F. and Fecht, F. (2012). Relationship lending in the interbank market and the price of liquidity. Discussion Papers, Deutsche Bundesbank, 22.

Brownlees, C. and Engle, R. (2010). Volatility, correlation and tails for systemic risk measurement. Working Paper Series, Department of Finance, $N Y U$.

Castro, C. and Ferrari, S. (2014). Measuring and testing for the systemically important financial institutions. Journal of Empirical Finance, 25:1-14.

Craig, B. and von Peter, G. (2014). Interbank tiering and money center banks. Journal of Financial Intermediation, 23:322-347.

Dungey, M., Luciani, M., and Veredas, D. (2013). Googling SIFIs. Systemic Risk: Liquidity Risk, Governance and Financial Stability, http://ssrn.com/abstract=2166504.

ECB (2009). The concept of systemic risk. European Central Bank Financial Stability Review, December.

ECB (2010). Analytical models and tools for the identification and assessment of systemic risks. European Central Bank Financial Stability Review, June.

ECB (2011). Systemic risk methodologies. European Central Bank Financial Stability Review, June.

Georg, C. (2013). The effect of the interbank network structure on contagion and financial stability. Journal of Banking and Finance, 77(7):2216-2228.

Giglio, S., Kelly, B., and Pruitt, S. (2015). Systemic risk and macroeconomy: An empirical evaluation. Journal of Financial Economics. forthcoming.

Glasserman, P. and Young, H. P. (2015). How likely is contagion in financial networks? Journal of Banking and Finance, 50:383-399.

Moreno, M. and Pena, J. (2013). Systemic risk measures: The simpler the better? Journal of Banking and Finance, 37:18171831 
Nucera, F., Schwaab, B., Koopman, S., and Lucas, A. (2015). The information in systemic risk rankings. SYRTO Working Paper Series, 20.

Tarashev, N., Borio, C., and Tsatsaronis, K. (2010). Attributing systemic risk to individual institutions. BIS workingpaper, nr. 308.

White, H., Kim, T.-H., and Manganelli, S. (2015). VAR for VaR: Measuring tail dependence using multivariate regression quantiles. Journal of Econometrics, 187:169-188. 